\title{
Importancia de la adherencia al tratamiento periodontal en pacientes diabéticos y el impacto del desapego.
}

\author{
Importance of adherence to periodontal treatment in \\ diabetic patients and the impact of detachment.
}

\author{
Arlen Marisol Lobato-García, * ${ }^{\star}$ Gustavo Tenorio-Torres, ${ }^{\ddagger}$ Alfredo Garcilazo-Gómez, ${ }^{\ddagger}$ \\ Karla Eugenia Miguelena-Muro, ${ }^{\ddagger}$ María Isabel de Fátima Luengas-Aguirre ${ }^{\ddagger}$
}

\section{RESUMEN}

La diabetes mellitus es una de las enfermedades sistémicas con mayor prevalencia en la población mundial. Este padecimiento representa uno de los factores de riesgo más importantes asociados con la enfermedad periodontal. El paciente con enfermedades crónicas como la diabetes necesita una disciplina convencida diaria y prolongada a través del tiempo (años) como única manera de reducir el impacto sistémico que deteriora la calidad de vida. Cuando se adhiere al tratamiento es posible observarlo en su condición estomatológica; sin embargo, cuando no se logra, se genera un deterioro en las condiciones bucales, principalmente enfermedad periodontal. En este artículo se presentan dos casos clínicos en los cuales se puede observar la mejoría de las condiciones bucales una vez que el paciente decide adherirse a su tratamiento. En el primero se aprecian las manifestaciones de manera notoria de un desapego en años posteriores. Se debe tener en cuenta que la adhesión al tratamiento no sólo depende de factores concernientes al paciente, también intervienen factores asociados al medio ambiente, situación económica, interacción médico-paciente, entre otros, por lo que resulta importante incentivar continuamente y acompañar al paciente en su tratamiento, así como conocer y entender la problemática de una enfermedad sistémica.

Palabras clave: Enfermedad periodontal, adhesión al tratamiento, diabetes mellitus.

\section{ABSTRACT}

Diabetes mellitus is one of the most prevalent systemic diseases in the world population. This condition represents one of the most important risk factors associated with periodontal disease. The patient with chronic diseases such as diabetes needs a daily and prolonged convinced discipline over time (years), as the only way to reduce the systemic impact, which deteriorates the quality of his life: when he has adherence to treatment it is possible to observe it in his stomatological condition, however when it is not achieved, a deterioration in the oral conditions is generated, mainly periodontal disease. This article presents two clinical cases where the improvement of oral conditions can be observed once the patient decides to adhere to their treatment. In the first one, the manifestations of a detachment in later years are noticeable. It should be taken into account that adherence to treatment does not only depend on factors concerning the patient. Also involved are factors associated with the environment, economic situation, doctorpatient interaction, among others, so it is important to continually encourage and accompany the patient in their treatment, as well as to know and understand the problem of a systemic disease.

Keywords: Periodontal disease, treatment adherence, diabetes mellitus.

\section{WWW.medigraphic.org.mx}

\footnotetext{
* Especialista en Periodoncia.

‡ División de Ciencias Biológicas y de la Salud. Departamento de Atención a la Salud. Departamento de Estomatología. Universidad Autónoma Metropolitana. México.
}

Recibido: 03 de octubre de 2019. Aceptado: 30 de abril de 2021.

Citar como: Lobato-García AM, Tenorio-Torres G, Garcilazo-Gómez A, Miguelena-Muro KE, Luengas-Aguirre MIF. Importancia de la adherencia al tratamiento periodontal en pacientes diabéticos y el impacto del desapego. Rev ADM. 2021; 78 (4): 221-228. https://dx.doi.org/10.35366/101077

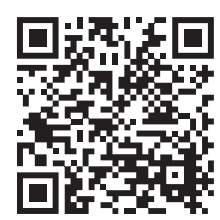




\section{INTRODUCCIÓN}

L a diabetes mellitus (DM) es una de las enfermedades sis_témicas con mayor prevalencia en la población mundial y a su vez es considerada un problema de salud pública. ${ }^{1}$

La diabetes corresponde a un grupo de enfermedades metabólicas caracterizadas por el mantenimiento de una condición de hiperglicemia que puede tener su origen en defectos en la secreción de insulina, en alteraciones de la acción de la insulina sobre las células blanco o en ambos procesos. ${ }^{2}$

La hiperglicemia crónica que ocurre en la diabetes está asociada con daño crónico y disfunción de diversos órganos y tejidos, tales como ojos, riñones, nervios, corazón, vasos sanguíneos y dentición. ${ }^{2}$ Entre las complicaciones tardías asociadas con la diabetes mellitus se ha señalado la enfermedad periodontal (EP). ${ }^{3}$

Se ha comprobado que la asociación entre las dos afecciones es bidireccional, es decir, no sólo la DM aumenta el riesgo de padecer EP, sino que estas últimas pueden dañar el control de la glucemia. ${ }^{4}$ La pérdida de inserción periodontal parece estar estrechamente vinculada al control metabólico de la diabetes. Los mecanismos que explican esta relación bidireccional son complejos. El sistema inmunitario participa activamente en la patogénesis de la DM, sus complicaciones y en la fisiopatología de la EP. Esta activación está principalmente relacionada con la vía de las citoquinas, que también poseen una función central en la respuesta del huésped frente al biofilm mi- crobiano periodontal. ${ }^{5}$ Es así como un mal control de esta enfermedad, medida a través de los niveles plasmáticos de hemoglobina glicosilada (HbA1c), se asoció con mayor prevalencia, severidad y extensión de la EP. ${ }^{2}$

La EP se convierte en factor de riesgo de la DM porque en ella la secreción de productos finales de glucosilación avanzados (AGE, siglas del término en inglés) se unen con receptores de membranas de células fagocíticas (neutrófilos y macrófagos) y sobrerregulan las funciones de los mediadores químicos proinflamatorios que mantienen una hiperglucemia crónica, tal como ocurre en la diabetes mellitus. Ambas entidades clínicas poseen factores genéticos y alteraciones microbiológicas e inmunológicas en común. La evidencia es que los niveles de glucosa se estabilizan después del tratamiento periodontal y la EP produce desestabilización de la glucemia. ${ }^{4}$

La periodontitis puede iniciar o aumentar la resistencia a la insulina de una manera similar a como lo hace la obesidad, favoreciendo la activación de la respuesta inmunológica sistémica iniciada por las citoquinas. La inflamación crónica generada por la liberación de estos mediadores de la inflamación está asociada con el desarrollo de la resistencia a la insulina, que además está condicionada por factores ambientales (pero modificados por la genética) como la escasa actividad física, la alimentación inadecuada, la obesidad o las infecciones. ${ }^{5}$

El control metabólico constituye un factor fundamental en el mantenimiento de la EP en los pacientes diabéticos. $^{5}$
Figura 1:

A-B) Ingreso a la clínica con agrandamiento gingival de seis meses de evolución. C)

Ortopantomografía. Se observa pérdida ósea generalizada.

D) Radiografías tomadas con radiovisiógrafo de zona anterior superior que destacan la pérdida ósea en dientes pilares de prótesis

parcial fija (PPF) OD 12 a 21.
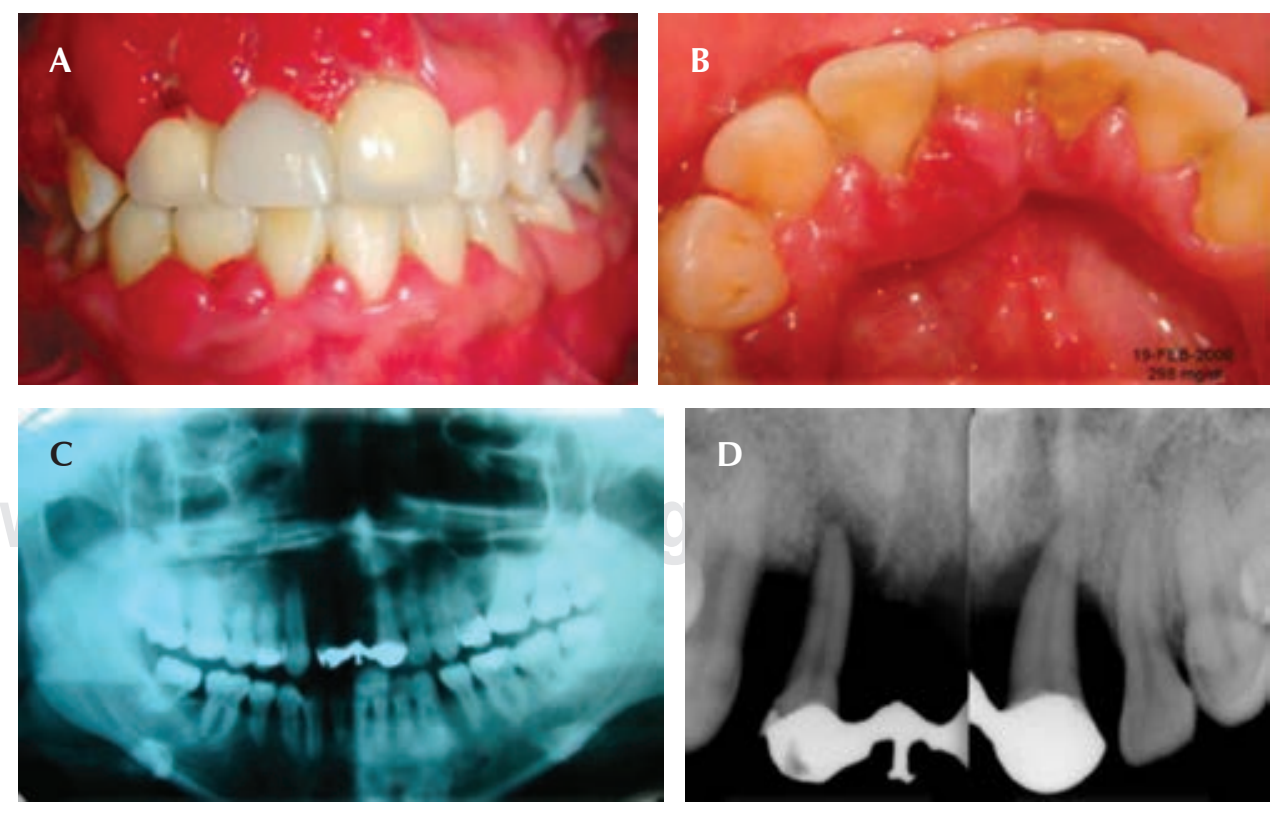

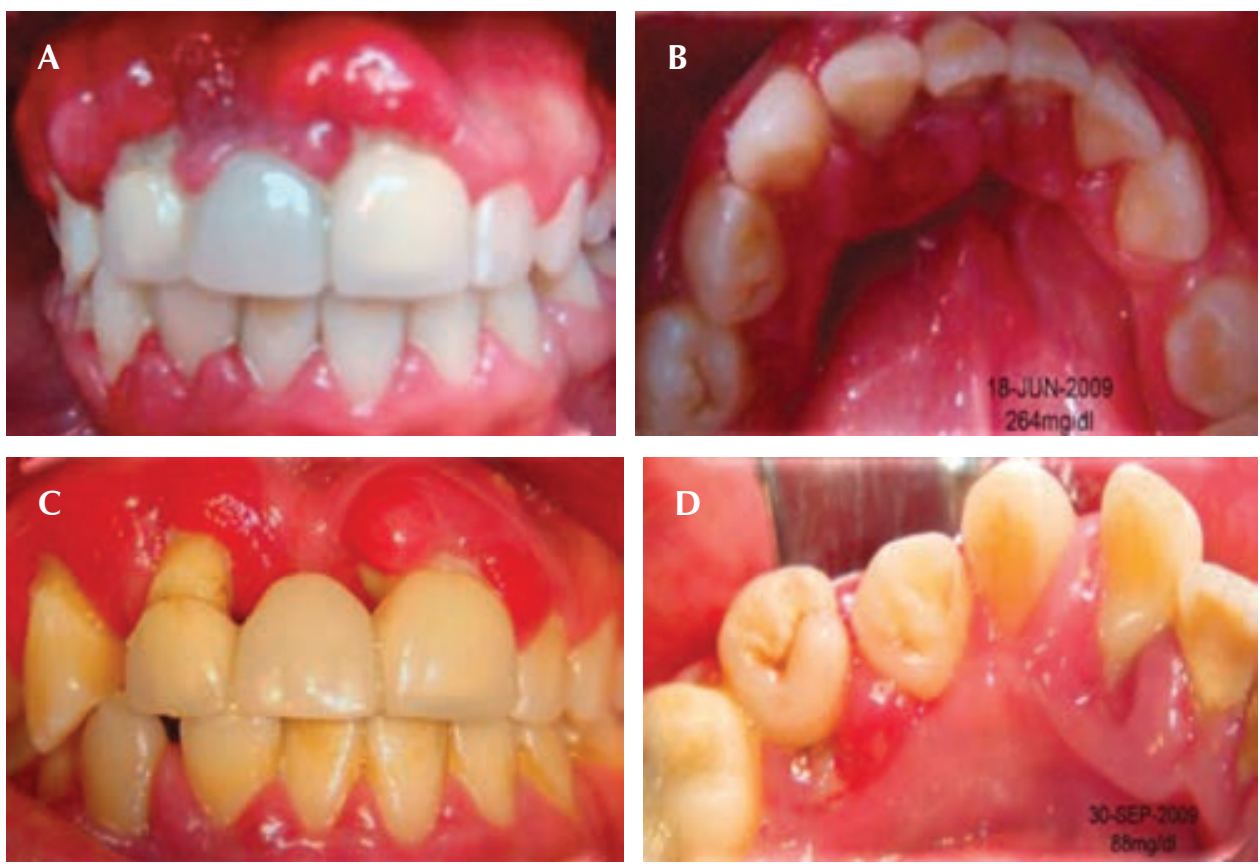

Figura 2:

A-B) Control mecánico y químico con clorhexidina a un mes de inicio de tratamiento. C-D)

Después de cuatro meses de inicio de fase I periodontal se logró reducir el agrandamiento gingival y controlar los niveles de glicemia.

El tratamiento general de los pacientes con enfermedad periodontal puede dividirse en tres fases:

1. Fase inicial o etiológica, encaminada a eliminar la infección y detener la progresión de la destrucción de los tejidos periodontales.

2. Fase correctiva, encaminada a restaurar la función y la estética.

3. Fase de mantenimiento, encaminada a prevenir la recurrencia de caries y de la enfermedad periodontal y a instaurar en el paciente una serie de medidas preventivas personales con el fin de evitar dicha reinfección. ${ }^{6}$

Se define como adherencia o apego al tratamiento al grado en el que el comportamiento y la actitud de una persona son congruentes con las recomendaciones médicas y del equipo de salud, con gran convencimiento individual, y que éstas se lleven cabo adecuadamente a través del tiempo.?

Entre las causas de la falta de apego al tratamiento de la diabetes mellitus se encuentra el desconocimiento de la enfermedad, baja escolaridad, situación económica, estados alterados de ánimo, deterioro sensorial, intolerancia al medicamento, y tratamientos alternativos. ${ }^{8}$

La evolución favorable de la diabetes exige la autorregulación de la conducta de salud, lo cual implica hacer esfuerzos personales para la búsqueda de estrategias que beneficien la adherencia a las indicaciones médicas, lo cual habla de la participación activa del paciente o implicación personal en el proceso de ajuste a la enfermedad. ${ }^{9}$

\section{PRESENTACIÓN DEL CASO}

Caso 1. Paciente femenino de 22 años de edad, refiere padecer diabetes mellitus (DM) tipo 1 desde hace dos años, «controlada» con insulina de acción intermedia.

Como antecedentes gineco-obstétricos, la paciente refiere tomar levonorgestrel (pastilla de emergencia) desde hace dos años, por lo menos dos veces al mes.

Referente a sus antecedentes heredofamiliares, su madre, abuelos y tíos maternos padecen diabetes.

A la inspección clínica se observa agrandamiento gingival en la zona anterior superior (Figura 1A), múltiples abscesos periodontales (Figura 1B), presencia de biofilm microbiano, cálculo supragingival y subgingival. Durante el examen periodontal se encontraron profundidades al sondaje de 5, 7 y hasta $13 \mathrm{~mm}$ y movilidad grado II, en la evaluación radiográfica se observó reabsorción ósea generalizada (Figura 1C y D).

Posterior a la evaluación clínica y radiográfica se decidió realizar interconsulta con el Hospital General «Dr. Manuel Gea González» para confirmar diagnóstico sistémico y adecuar terapia, la paciente se diagnosticó como diabética tipo 2, modificando el medicamento, eliminando insulina y prescribiendo metformina con glibenclamida. 
Con los elementos obtenidos se realizó el diagnóstico periodontal:

- Periodontitis etapa IV generalizada grado C.

- Agrandamiento gingival crónico de tipo inflamatorio.

- Absceso periodontal agudo.

Una vez establecido el diagnóstico, se emitió el plan de tratamiento que consistió en realizar una fase I periodontal, alternando sus citas con el Hospital «Dr. Manuel Gea González» para el control de su glicemia. Después de modificar el tratamiento para la diabetes por parte del Hospital y realizando el control mecánico del biofilm microbiano, se comenzaron a observar cambios clínicos, como disminución del aumento de volumen (Figura 2A y C) y desaparición de los absceso periodontales (Figura $2 B$ y D). Una vez que los niveles de glucosa se estabilizaron, se inició la fase II del nivel de tratamiento periodontal (extracciones, desbridamiento por colgajo con injerto óseo), se medicó a la paciente con doxiciclina (previo al procedimiento quirúrgico una cápsula de 100 mg cada 12 horas, segundo día hasta el séptimo día, una cápsula de
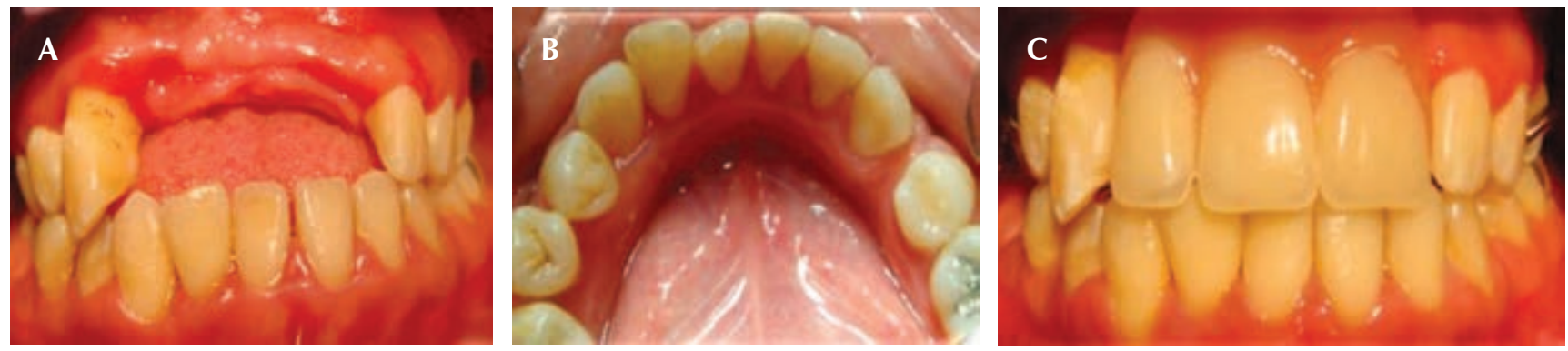

Figura 3: A-B) Se puede observar a la paciente tres meses después de haber realizado la fase II del tratamiento periodontal. C) Paciente con prótesis parcial removible.
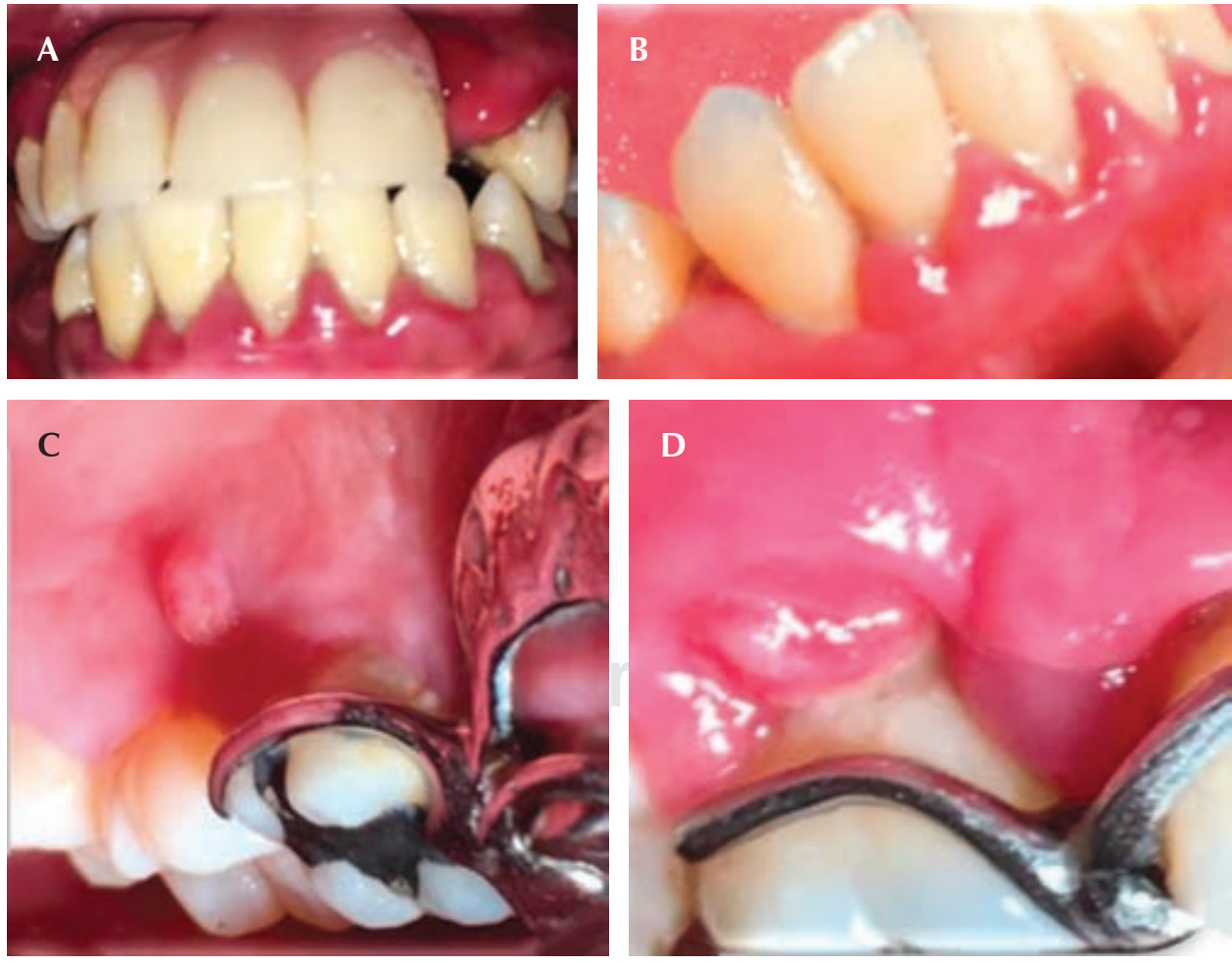

Figura 4:

A-D) Después de seis años la paciente regresó con reincidencia de enfermedad periodontal, A-B) se observó presencia de biofilm microbiano supragingival y subgingival, recesiones gingivales, agrandamiento gingival en zonas anteriores y posteriores bilaterales, C) presencia de absceso en OD 26. 

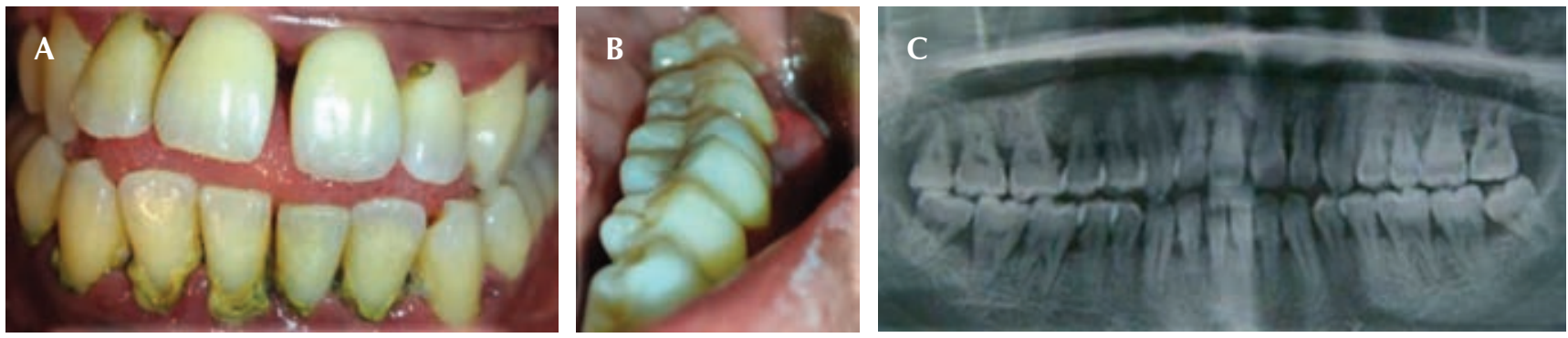

Figura 5: A) Ingreso a la clínica. Se observa cálculo supragingival. B) Presencia de absceso periodontal en zona de OD 37. C) Ortopantomografía en la que se observa pérdida ósea generalizada.

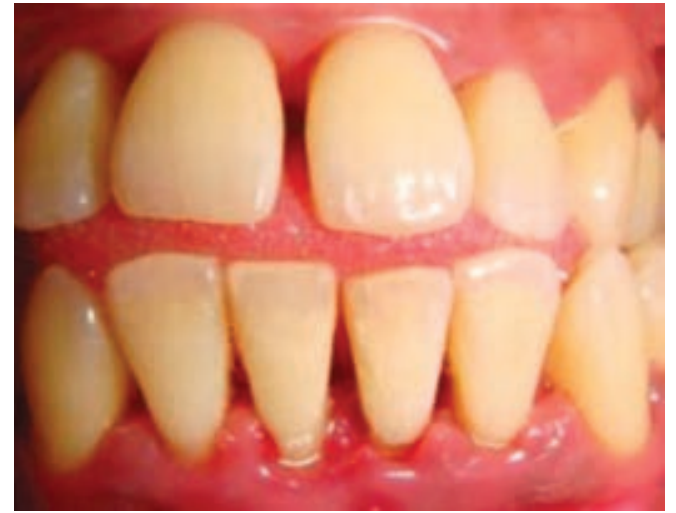

Figura 6: Se muestra la evolución del paciente después de haber realizado la fase I periodontal.

$50 \mathrm{mg}$ cada 12 horas) y ketorolaco de $10 \mathrm{mg}$, una tableta cada ocho horas o sólo en caso de dolor. Después de estabilizar periodontalmente a la paciente se incorporó a la terapia de mantenimiento y se dio inicio el tratamiento de rehabilitación, elaborando una prótesis parcial removible (Figura 3A-C). Después de dos años de adhesión al tratamiento, la paciente dejó de asistir a consulta.

Seis años después acude nuevamente, presentando las siguientes características: agrandamientos gingivales de tipo inflamatorio generalizados, presencia de biofilm microbiano, cálculo supragingival y subgingival, abscesos periodontales, recesiones gingivales, todo esto evidencia un desapego al mantenimiento periodontal. Los niveles de glucemia obtenidos por medio de la toma capilar fueron de $450 \mathrm{mg} / \mathrm{dL}$ (Figura $4 \mathrm{~A}-\mathrm{C}$ ).

Caso 2. Paciente masculino de 38 años de edad se presentó al servicio de emergencia, debido a molestia en la zona inferior izquierda, el paciente refirió ser «sano» y no padecer ninguna enfermedad. Dentro de sus an-
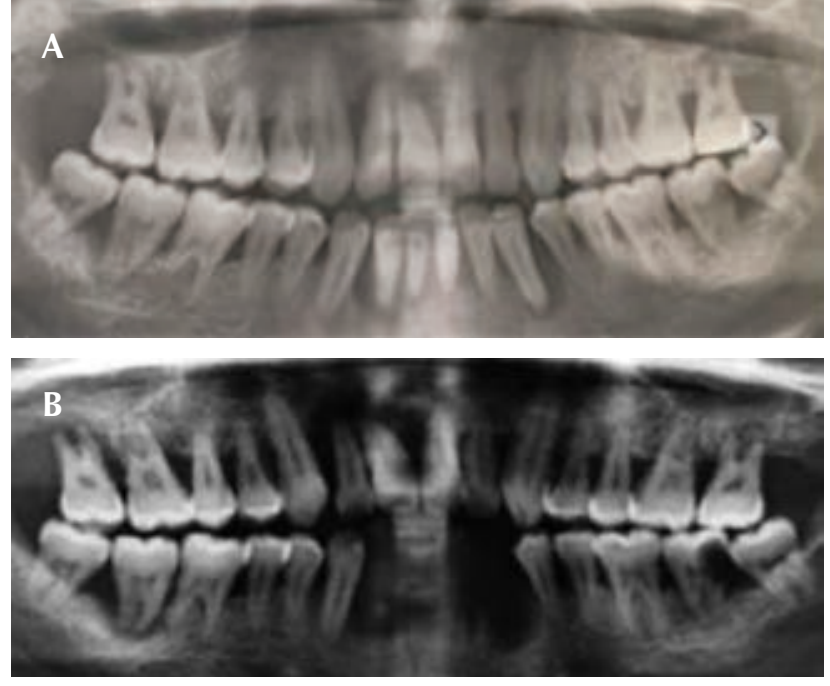

Figura 7: A) Ortopantomografía que demostró la pérdida ósea después de haber abandonado el tratamiento por dos años. B) Ortopantomografía en la que se observó la progresión de la enfermedad periodontal tres años después.

tecedentes heredofamiliares, su padre padece diabetes mellitus e hipertensión arterial. A la inspección física se determinó un índice de masa corporal (IMC), obesidad tipo II, tensión arterial de 140/90 mm/Hg. En el interrogatorio que corresponde a hábitos y toxicomanías, el paciente mencionó ser fumador.

A la inspección clínica se observaron múltiples abscesos periodontales, presencia de placa microbiana (biofilm), cálculo supragingival y subgingival (Figura 5). Con base en estas características y después de haber identificado diversos factores de riesgo, se tomó glucemia por medio de una prueba capilar con valor de $172 \mathrm{mg} / \mathrm{dL}$ en ayuno. Con los elementos obtenidos se realizó el diagnóstico periodontal: periodontitis etapa III generalizada grado B (Figura 6). 

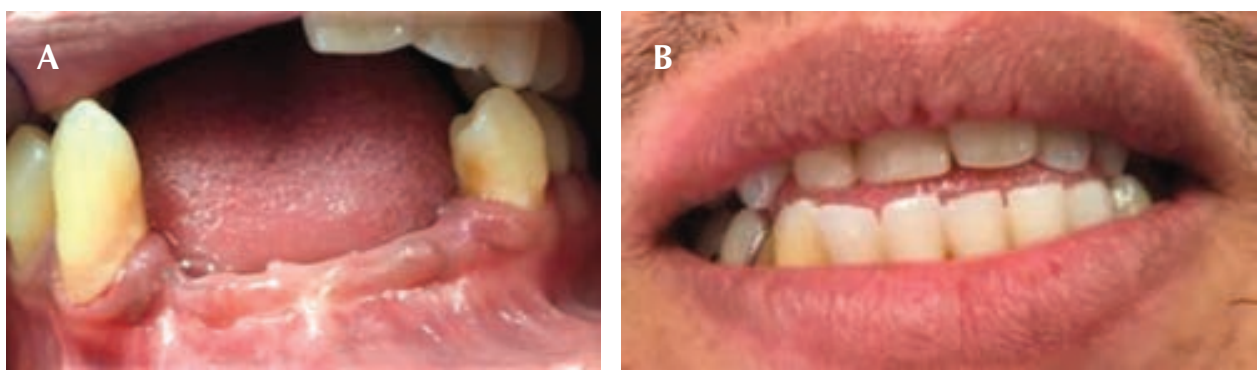

Figura 8:
A) Después del desapego al tratamiento periodontal, el paciente regresa con mayores problemas periodontales y pérdida de dientes. B) Se realizó prótesis parcial removible (PPR) inmediata provisional.

Se inició la fase I y con base en la adherencia al tratamiento periodontal y un adecuado seguimiento, a pesar de la renuencia de iniciar un tratamiento sistémico se obtuvo una respuesta favorable. El paciente abandona el tratamiento por dos años, a su regreso afirmó que ya es diabético e hipertenso, sometido a tratamiento a través de metformina y enalapril respectivamente. Por medio de radiografías panorámicas y la inspección clínica se observó mayor pérdida ósea generalizada, lo cual mostró la progresión de la enfermedad periodontal, debido al desapego al tratamiento y la suma del factor sistémico (Figura 7A y B). Se inició (nuevamente) la fase I periodontal, realizando múltiples extracciones, debido a las condiciones en las que se presentó. Posteriormente se rehabilitó por medio de una prótesis parcial removible provisional inmediata (Figura $8 A$ y $B$ ).

\section{DISCUSIÓN}

La Organización Mundial de la Salud (OMS) menciona que en los países desarrollados la adherencia a los tratamientos a largo plazo alcanza sólo 50\%, y supone que esta deficiencia sea aún mayor en países en desarrollo dada la escasez de recursos y las inequidades en el acceso a la atención sanitaria. ${ }^{10}$ Krass y colaboradores establecen que los factores principales asociados con la falta de adhesión son el estado mental, específicamente la depresión como consecuencia de los cambios metabólicos cerebrales ${ }^{7}$ y los costos de la medicación. ${ }^{11}$

Algunos estudios determinan la relación importante entre la adherencia terapéutica y el número de citas, sugiriendo que a mayor frecuencia de consultas mejor adherencia (Figura 9); ${ }^{12}$ en los casos clínicos presentados se hace evidente tal condición durante el tratamiento y mantenimiento de los pacientes diabéticos en el consultorio dental, por lo que las revisiones programadas deberán incluir control glucémico, educación sobre la higiene oral adecuada para evitar la acumulación de placa, la irritación de la mucosa y la aparición de candidiasis oral, además de la vigilancia en el ajuste y limpieza de las prótesis fijas, removibles o totales.

Es importante hacerle hincapié al paciente que la adhesión al tratamiento no sólo se refiere al cumplimiento de la toma de medicamentos, sino también a seguir una serie de indicaciones. Jiménez menciona que más de 95\% del cuidado de la diabetes es responsabilidad del paciente, ya que constantemente a lo largo de un día el/ la paciente debe realizar tareas de autocuidado y/o tomar decisiones relevantes con respecto a la enfermedad. ${ }^{13}$

Los resultados analizados por Hechavarría y asociados muestran la importancia que tienen las actividades de promoción de salud y la prevención de enfermedades en la atención al diabético. ${ }^{14}$

Cuando no se cuenta con un adecuado control y falta de adherencia al tratamiento de los pacientes diabéticos se presentan efectos a corto y largo plazo, impactando negativamente en la calidad de vida del paciente.

En los casos clínicos del presente artículo se puede observar recaída y mayor severidad ante la falta de la adherencia, tal y como lo menciona la OMS. ${ }^{10}$

Anderson y Glasgow reportan que existe una asociación positiva entre la participación del paciente en el diseño de su plan de tratamiento y las conductas de autocuidado de la diabetes, lo que provoca una adherencia al tratamiento, incluyendo cambios en el estilo de vida y mejoramiento en la calidad y esperanza de vida de estos pacientes. ${ }^{15,16}$

Considerando que la adherencia al tratamiento, como lo mencionan (Hoyos, Arteaga, Muñoz), también involucra factores asociados al paciente (cultura, creencias, nivel cognitivo y sensorial), factores asociados al ambiente (aislamiento social, mitos en salud), factores asociados al contexto económico (costos de la terapéutica, accesibilidad a los servicios de salud, estrato socioeconómico) y factores asociados con la interacción médico-paciente (duración de la consulta y claridad en las instrucciones dadas). El desencuentro con los discursos de los profesionales de la salud también está permeado por la cultura del 
regaño, por la prohibición y la imposición de esquemas, lo que se traduce en incumplimiento del tratamiento. ${ }^{7}$

En relación con la edad, existen datos que indican aparentemente mayor presencia de pacientes cumplidores en la medida en que se transita por la etapa de la adultez media hacia la tercera edad, que podrían estar influenciados por lo señalado en la literatura en cuanto a que a mayor edad el paciente puede cumplir más con las conductas de autocuidado, desde la creencia de que las personas a medida que van envejeciendo tienen un estilo de vida más regular en comparación con los más jóvenes. Estos resultados contradictorios refuerzan la idea de que la edad es un factor irregular en la explicación del fenómeno de la adherencia. ${ }^{17}$

\section{CONCLUSIONES}

La participación del odontólogo en la atención sistémica temprana de la diabetes es posible si el profesional tiene muy presente un protocolo de acción en caso de identificar factores de riesgo que promuevan o identifiquen el padecimiento de la diabetes, el control de la enfermedad periodontal y la adherencia a un tratamiento integral multidisciplinario rompe un círculo vicioso que favorece la posibilidad de mantener un equilibrio en los pacientes que padecen esta enfermedad, lamentablemente al pasar el tiempo el desapego al tratamiento bucal y sistémico es muy frecuente. Es común que el odontólogo juzgue como irresponsabilidad por parte del paciente el desinterés en el tratamiento; sin embargo, es importante conocer que la falta de adherencia no sólo depende de la variable del

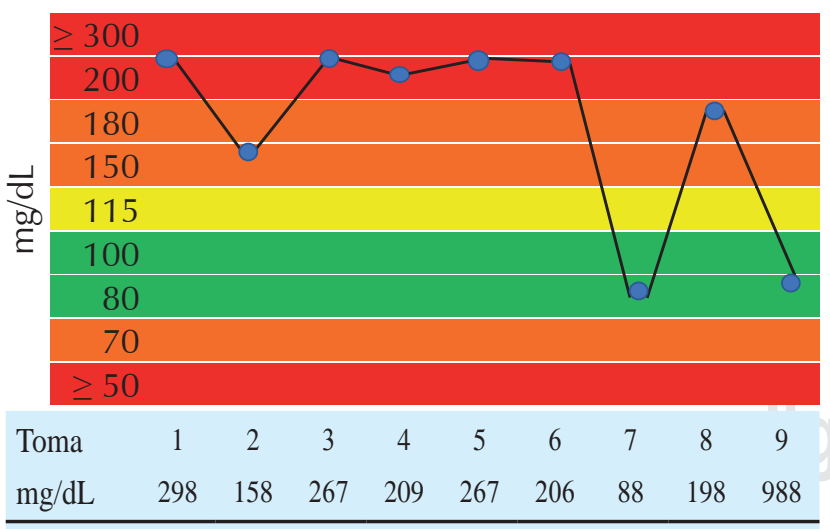

Toma $1298 \mathrm{mg} / \mathrm{dl}$, toma $2158 \mathrm{mg} / \mathrm{dl}$, toma $3267 \mathrm{mg} / \mathrm{dl}$, toma $4209 \mathrm{mg} / \mathrm{dl}$, toma 5 $267 \mathrm{mg} / \mathrm{dl}$, toma $6206 \mathrm{mg} / \mathrm{dl}$, toma $788 \mathrm{mg} / \mathrm{dl}$, toma $8198 \mathrm{mg} / \mathrm{dl}$, toma $988 \mathrm{mg} / \mathrm{dl}$.

Figura 9: Niveles de glucemia de la paciente del caso 1 en cada una de sus citas. paciente, sino que existen otros factores concernientes a la evolución y desarrollo de la diabetes, los cuales pueden crear diversas afecciones metabólicas, entre ellas en el cerebro, que impidan al paciente tomar buenas decisiones.

Diseñar estrategias que incorporen diversas especialidades, familia y el acompañamiento del odontólogo como parte de un equipo interdisciplinario a través del tiempo podría disminuir el riesgo del descuido o abandono del seguimiento y atención de la o las diversas enfermedades que comprometen la salud de cada paciente diabético.

A pesar de los avances diagnósticos y terapéuticos de las últimas décadas en el control de la DM es difícil resolver el problema relacionado con el cumplimiento o adherencia, pues el paciente es autónomo y toma su propia decisión de la necesidad de tomar la medicación, y realizar los cambios necesarios en el estilo de vida de acuerdo con su propia percepción, conocimiento y preocupación por la enfermedad. Muchas de las indicaciones terapéuticas no pueden ser supervisadas regularmente por personas ajenas al paciente y por el contrario, quedan bajo su directa responsabilidad, entonces es el paciente quien en definitiva decide si debe cumplir o no.

En el caso de la enfermedad diabetes mellitus 2, que es el de interés en este artículo, se ha observado que las implicaciones directas e indirectas de la misma afectan el pensamiento, las emociones y la conducta de las personas, impactando seriamente la calidad de vida de nuestros pacientes.

Es claro, sin embargo, que para lograr una visión clara y eficiente de la medicina bucal para el cuidado de pacientes con enfermedades sistémicas como en este caso la DM2 se requieren cambios en la filosofía de la práctica de la estomatología, donde los prestadores de este servicio se conciban como profesionales estrategas y planificadores de la salud bucal integral. Esta nueva filosofía será imposible sin una discusión profunda sobre la formación de prestadores de servicios e investigadores en el campo de la estomatología.

\section{REFERENCIAS}

1. Miranda GM, Montoya ZYP, Saldarriaga SA. Diabetes y enfermedad periodontal: hacia un modelo clínico bidireccional. Revista Nacional de Odontología. 2012; 8: 76-87.

2. Smith P, Retamal I, Cáceres M, Romero A, Silva D, Arancibia R et al. Diabetes y su impacto en el territorio periodontal. Rev Clin Periodoncia Implantol Rehabil Oral. 2012; 5 (2): 90-92.

3. Arrieta Blanco JJ, Bartolomé Villar B, Jiménez Martínez E, Saavedra Vallejo P, Arrieta Blanco FJ. Problemas bucodentales en pacientes con diabetes. Med Oral. 2003; 8 (2): 97-109.

4. Fajardo Puig ME, Rodríguez Reyes O, Hernández Cunill M, Mora Pacheco N. Diabetes mellitus y enfermedad periodontal: aspectos 
fisiopatológicos actuales de su relación. MEDISAN. 2016; 20 (6): 845-850.

5. Herrera D, Rodríguez Casanovas HJ, Herrera Pombo JL. Diabetes y enfermedades periodontales. Madrid: SEPA/Fundación SED; 2015 [citado el 26 de enero de 2016]. Disponible en: http://www.sepa.es/ images/stories/SEPA/ESTAR_al_DIA/DIABETES/Dossier_DIABETES. pdf

6. Sanz-Sánchez I, Bascones-Martínez A. Terapéutica periodontal de mantenimiento. Av Periodon Implantol. 2017; 29 (1): 11-21.

7. Hoyos TN, Arteaga MV, Muñoz M. Factores de no adherencia al tratamiento en personas con diabetes mellitus tipo 2 en el domicilio. La visión del cuidador familiar. Invest Educ Enferm. 2011; 29 (2): 194-203.

8. Durán-Varela BR, Rivera-Chavira B, Franco-Gallegos E. Apego al tratamiento farmacológico en pacientes con diagnóstico de diabetes mellitus tipo 2. Salud Pública Méx. 2001; 43 (3): 233-236.

9. Castillo MM, Martín AL, Almenares RK. Therapeutic adherence and influencing factors in patients with type 2 diabetes mellitus. Rev Cubana Med Gen Integr. 2017; 33 (4): 1-10.

10. Organización Mundial de la Salud. Adherencia a los tratamientos a largo plazo. Pruebas para la acción. Ginebra: OMS; 2004 [citado el 7 de septiembre del 2019]. Disponible en: https://www.paho.org/ hq/dmdocuments/2012/WHO-Adherence-Long-Term-TherapiesSpa-2003.pdf

11. Krass I, Schieback P, Dhippayom T. Adherence to diabetes medication: a systematic review. Diabet Med. 2015; 32 (6): 725-737.

12. Orozco-Beltrán D, Mata-Cases M, Conthe P, Mediavilla J, Miranda C. Abordaje de la adherencia en diabetes mellitus tipo2: situación actual y propuesta de posibles soluciones. Aten Primaria. 2016; 48 (6): 406-420.

13. Jiménez Chafey MI, Dávila M. Psicodiabetes. Av Psicol Latinoam [Internet]. 2007 [consultado el 27 de agosto de 2019]; 25 (1): 126-143. Disponible en: http://www.scielo.org.co/scielo.php?pid=S1794$47242007000100012 \&$ script $=$ sci_abstract $\&$ tlng $=$ es

14. Hechavarría Martínez BO, Núñez Antúnez L, Fernández Toledo M, Cobas Pérez N. Principales alteraciones bucodentales en pacientes con diabetes mellitus. MEDISAN. 2016; 20 (9): 2062-2068.

15. Anderson R, Funnell M, Carlson A, Saleh-Statin N, Cradock S, Chas Skinner T. Facilitating self-care through empowerment. In: Snoek F, Chas Skinner T (Eds.). Psychology in diabetes care. London: Wiley. Psychology in Diabetes Care; 2000. pp. 71-97.

16. Glasgow RE, Fisher EB, Anderson BJ, LaGreca A, Marrero D, Johnson SB et al. Behavioral science in diabetes. Contributions and opportunities. Diabetes Care. 1999; 22 (5): 832-843.

17. Ramos RY, Morejón SR, Gómez VM, Reina SM, Rangel DC, Cabrera MY. Adherencia terapéutica en pacientes con diabetes mellitus tipo 2. Rev Finlay. 2017; 7 (2): 89-98.

Correspondencia: Alfredo Garcilazo-Gómez

E-mail: alfredogarcilazo@yahoo.com.mx

Conflicto de intereses: Los autores declaran no tener ningún conflicto de intereses.

Financiamiento: Ninguno. 